\title{
Microwave-Assisted Parallel Synthesis of a 2-Aryl-1H-Isoindole-1,3-Dione Library
}

\author{
Bernardo M. Barchín, Ana M. Cuadro, Julio Alvarez-Builla* \\ Departamento de Química Orgánica, Universidad de Alcalá, Ctra. Madrid-Barcelona Km 33,600, C.P. 28871, Alcalá de Henares, Madrid, \\ Spain
}

Fax +34(91)8854686; E-mail: julio.alvarez@uah.es

Received 25 October 2001

Abstract: An efficient parallel synthesis of a representative 28member library of phthalimides is described. Parallel chromatography afforded the library members in suitable purity and with high yields.

Key words: library, microwave, phthalimide, 2 -aryl- $1 H$-isoindole1,3-dione, TNF- $\alpha$

Imide derivatives are compounds of considerable interest due to their biological properties, ${ }^{1}$ and their interest as general intermediates in synthesis ${ }^{2}$ and in polymer chemistry. ${ }^{3}$ Today, available routes for the synthesis of imide derivatives involve either Lewis-acid mediated condensation of an amine with maleic or phthalic anhydride ${ }^{2 a, 4}$ or $\mathrm{N}$-alkylation of the corresponding imide with halides, ${ }^{5}$ or alcohols under Mitsunobu conditions. ${ }^{2 a, 4 a}$ There is, however, still need of simple, efficient and general methods for the synthesis of functionalised imides. Recently, a substantial improvement of classical methods has been obtained using microwaves ${ }^{6}$ and solvent-free procedures with $^{7}$ and without ${ }^{8}$ catalyst, have been published.

In an effort to develop an efficient one-pot chemical transformation, we had developed a method for a rapid parallel ${ }^{9}$ synthesis of small libraries, using a domestic microwave oven, and we report the application of the technique to the synthesis of a phthalimide library, as a technique capable of providing a large numbers of interesting intermediates.

The procedure has been developed along several steps:

i) Structure of the Oven. Highest Irradiation Area Determination. In a domestic oven, not all areas in the dish are irradiated with the same intensity. It is thus, necessary to fix, for reproducibility, where the samples should be placed. The determination of the highest irradiation area (HIA) -which is similar for nearly all commercial domestic ovens- was easily performed by heating a disk of moist filter paper ( $26 \mathrm{~cm}$ diameter) placed over the oven dish, for $15 \mathrm{~min}$ ( $550 \mathrm{~W}$ output). As a result, a burned area between $16 \mathrm{~cm}$ and $22 \mathrm{~cm}$ from the central point of the circle, was obtained. Consequently, a disk made of teflon (5 $\mathrm{mm}$ thick) was designed (Figure 1, measures in $\mathrm{mm}$ ), able to hold 28 vials ( $20 \mathrm{~mm}$ diameter) in the area indicated.

Synlett 2002, No. 2, 0102 2002. Article Identifier:

1437-2096,E;2002,0,02,0343,0345,ftx,en;G31501ST.pdf.

(1) Georg Thieme Verlag Stuttgart - New York

ISSN 0936-5214
Other similar devices for a smaller number of bigger samples are currently being tested.

ii) Optimal Reaction Conditions. The reaction between $p$ anisidine and phthalic anhydride was chosen as a model process, and tests were carried using open vials in the teflon disk -evenly spaced on it- at different oven outputs and reaction times. With the experiments, it was concluded that one sample, irradiated $1 \mathrm{~min}$ at $550 \mathrm{~W}$ in the HIA, produced the highest yields. Also, it was concluded that the sample, once the product was formed, could be additionally heated up to $15 \mathrm{~min}$ at the same energy, without appreciable decomposition.

The optimal reaction conditions ( $1 \mathrm{~min} 550 \mathrm{~W}$ ) were applied to four anilines: $\left(a, R^{3}=O m e ; b, R^{1}=R^{5}=E t ; c, R^{1}\right.$ $=\mathrm{Me}, \mathrm{R}^{2}=\mathrm{Cl} ; \mathrm{d}, \mathrm{R}^{3}=\mathrm{F}$ ) each test being repeated four times, and giving the following mean yields: a) $95 \pm$ $0.28 \%$; b) $78 \pm 2.98 \%$,c) $80 \pm 2.70 \%$; d) $80 \pm 2.85 \%$.

In a typical general procedure, $4 \mathrm{~mL}$ vials, containing a mixture of the corresponding phthalic anhydride $(0.68$ $\mathrm{mmol})$ and the aniline $(0.68 \mathrm{mmol})$, without solvent, were irradiated in a domestic microwave oven ${ }^{10}$ at $550 \mathrm{~W}$ for $12-15 \mathrm{~min}$. Reaction mixtures were diluted in $1-3 \mathrm{~mL}$ of $\mathrm{CH}_{2} \mathrm{Cl}_{2}-\mathrm{MeOH}(90: 10)$ and filtered through a $2 \mathrm{~cm}$ pad of neutral alumina, using a VacMaster SPE sample processing station. ${ }^{11}$ The vials with the organic extracts were evaporated $^{12}$ to leave the crude products which were purified by recrystalilsation when needed.

iii) Time Versus Number of Samples: Starting from p-anisidine and phthalic anhydride, sets of 4,8 or 12 samples were irradiated with different reaction times (Table 1).

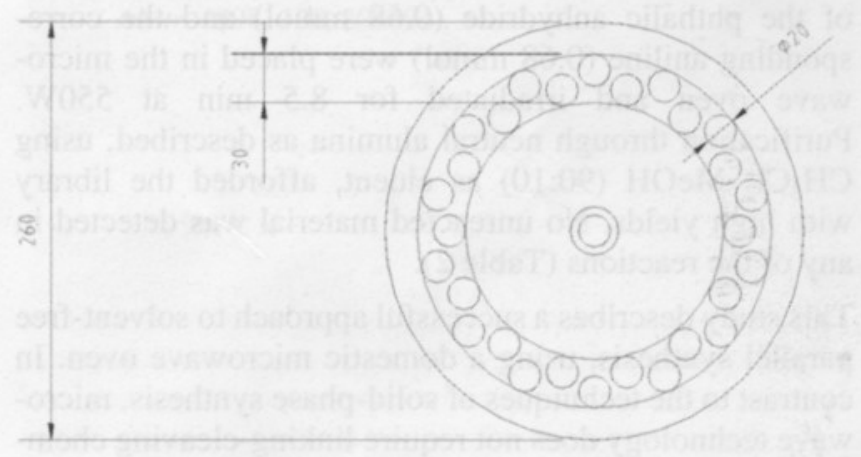

Figure 1 
From these results, the time required to obtain yields around $95 \%$ or higher were determined, and the time versus number of samples was plotted ${ }^{13}$ and fitted to a curve as indicated in Figure 2. The model serves, as a reference, to calculate the time in minutes ( $t$ ) needed to expand the conditions developed for one sample, to a set of a given number of vials (n) (Equation).

Table 1 Time Versus Number of Samples

\begin{tabular}{llll}
\hline $\mathrm{N}^{\circ}$ samples & Time $(\mathrm{min})$ & Time/sample & MeanYield $(\%)$ \\
\hline 1 & 1 & 1 & $95 \pm 0.28$ \\
4 & 3 & 0.75 & $96 \pm 0.36$ \\
8 & 5 & 0.625 & $96 \pm 1.43$ \\
12 & 6 & 0.5 & $96 \pm 4.63$ \\
\hline
\end{tabular}

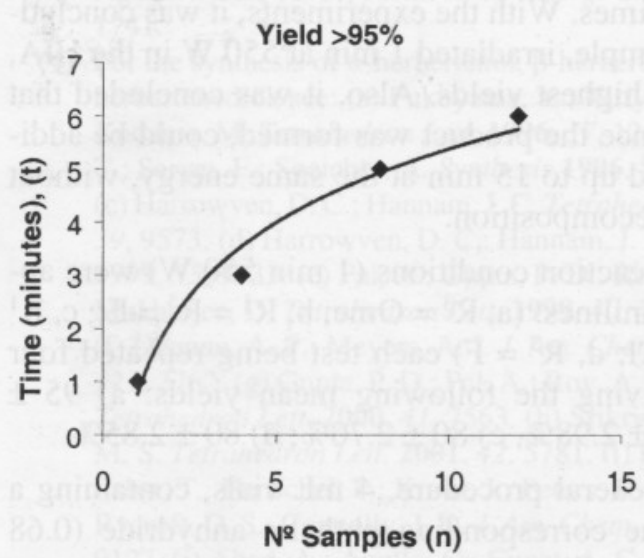

Figure 2

$$
\mathrm{t}=2.004 \operatorname{Ln}(\mathrm{n})+0.7687
$$

$\mathrm{r}^{2}=0.9715 ; \mathrm{s}=0.32 *$

$\mathrm{r}^{2}=$ correlation coefficient $\mathrm{s}=$ standard error

\section{Equation}

iv) Parallel Synthesis of N-Phenylphthalimides: The above mentioned model was applied to the synthesis of a library of 28 phthalimides (Scheme), giving a $t=7.5 \mathrm{~min}$. One additional minute was added to favour the less reactive anilines. Consequently, 28 vials containing a mixture of the phthalic anhydride $(0.68 \mathrm{mmol})$ and the corresponding aniline $(0.68 \mathrm{mmol})$ were placed in the microwave oven and irradiated for $8.5 \mathrm{~min}$ at $550 \mathrm{~W}$. Purification through neutral alumina as described, using $\mathrm{CH}_{2} \mathrm{Cl}_{2}-\mathrm{MeOH}$ (90:10) as eluent, afforded the library with high yields. No unreacted material was detected in any of the reactions (Table 2).

This study describes a successful approach to solvent-free parallel synthesis, using a domestic microwave oven. In contrast to the techniques of solid-phase synthesis, microwave technology does not require linking-cleaving chemistry and can produce compounds immediately available for biological screening.
Table 2 Synthesis of 2-Aryl-(5-methyl)-1H-isoindole-1,3-(2H)-diones $^{\mathrm{a}}$<smiles>[R]c1ccc2c(c1)C(=O)N(c1[Y]([H])c([R7])c([R])c([R7])c1[2H])C2=O</smiles>

\begin{tabular}{|c|c|c|c|c|c|c|c|c|}
\hline Entry & Anh. & $\mathrm{X}$ & $\mathrm{R}^{\prime}$ & $\mathrm{R}^{2}$ & $\mathrm{R}^{3}$ & $\mathrm{R}^{4}$ & $\mathrm{R}^{5}$ & $\begin{array}{l}\text { Comp. } \\
\text { ( } \% \text { yield) }\end{array}$ \\
\hline 1 & 1 & $\mathrm{C}$ & $\mathrm{H}$ & $\mathrm{H}$ & $\mathrm{H}$ & $\mathrm{H}$ & $\mathrm{H}$ & 3a $(90)^{14}$ \\
\hline 2 & 1 & $\mathrm{C}$ & $\mathrm{H}$ & $\mathrm{H}$ & $\mathrm{OMe}$ & $\mathrm{H}$ & $\mathrm{H}$ & 3b $(97)^{15}$ \\
\hline 3 & 1 & $\mathrm{C}$ & $\mathrm{OMe}$ & $\mathrm{H}$ & $\mathrm{H}$ & $\mathrm{H}$ & $\mathrm{H}$ & 3c (93) \\
\hline 4 & 1 & $\mathrm{C}$ & $\mathrm{H}$ & $\mathrm{CF}_{3}$ & $\mathrm{H}$ & $\mathrm{H}$ & $\mathrm{H}$ & 3d $(91)^{16}$ \\
\hline 5 & 1 & $\mathrm{C}$ & $\mathrm{H}$ & $\mathrm{H}$ & $i$-Pr & $\mathrm{H}$ & $\mathrm{H}$ & $3 e(71)^{17}$ \\
\hline 6 & 1 & $\mathrm{C}$ & $\mathrm{Et}$ & $\mathrm{H}$ & $\mathrm{H}$ & $\mathrm{H}$ & Et & 3f $(78)^{14}$ \\
\hline 7 & 1 & C & $\mathrm{F}$ & $\mathrm{H}$ & $\mathrm{H}$ & $\mathrm{H}$ & $\mathrm{H}$ & $\mathbf{3 g}(87)^{18}$ \\
\hline 8 & 1 & C & $\mathrm{H}$ & $\mathrm{H}$ & $\mathrm{F}$ & $\mathrm{H}$ & $\mathrm{H}$ & 3h $(93)^{18}$ \\
\hline 9 & 1 & $\mathrm{C}$ & $\mathrm{Me}$ & $\mathrm{H}$ & $\mathrm{H}$ & $\mathrm{H}$ & $i-\operatorname{Pr}$ & $\mathbf{3 i}(95)^{14}$ \\
\hline 10 & 1 & C & $i-\operatorname{Pr}$ & $\mathrm{H}$ & $\mathrm{H}$ & $\mathrm{H}$ & $i-\operatorname{Pr}$ & $\mathbf{3 j}(90)^{14}$ \\
\hline 11 & 1 & C. & $\mathrm{H}$ & $\mathrm{Cl}$ & $\mathrm{H}$ & $\mathrm{H}$ & $\mathrm{H}$ & $\mathbf{3 k}(93)^{19}$ \\
\hline 12 & 1 & $\mathrm{C}$ & $\mathrm{H}$ & $\mathrm{H}$ & $\mathrm{Cl}$ & $\mathrm{H}$ & $\mathrm{H}$ & $3 \mathbf{l}(95)^{15}$ \\
\hline 13 & 1 & C & $\mathrm{Br}$ & $\mathrm{H}$ & $\mathrm{Br}$ & $\mathrm{H}$ & $\mathrm{CO}_{2} \mathrm{Me}$ & $3 \mathbf{m}(34)$ \\
\hline 14 & 1 & $\mathrm{C}$ & $\mathrm{Me}$ & $\mathrm{Cl}$ & $\mathrm{H}$ & $\mathrm{H}$ & $\mathrm{H}$ & 3n (93) \\
\hline 15 & 1 & $\mathrm{~N}$ & - & $\mathrm{H}$ & $\mathrm{Br}$ & $\mathrm{H}$ & - & 3o $(43)^{20}$ \\
\hline 16 & 2 & $\mathrm{C}$ & $\mathrm{H}$ & $\mathrm{H}$ & $\mathrm{H}$ & $\mathrm{H}$ & $\mathrm{H}$ & $4 \mathbf{a}(65)^{21}$ \\
\hline 17 & 2 & C & $\mathrm{H}$ & $\mathrm{H}$ & $\mathrm{OMe}$ & $\mathrm{H}$ & $\mathrm{H}$ & 4b (89) \\
\hline 18 & 2 & C & $\mathrm{OMe}$ & $\mathrm{H}$ & $\mathrm{H}$ & $\mathrm{H}$ & $\mathrm{H}$ & $4 c(87)$ \\
\hline 19 & 2 & C & $\mathrm{H}$ & $\mathrm{CF}_{3}$ & $\mathrm{H}$ & $\mathrm{H}$ & $\mathrm{H}$ & 4d (88) \\
\hline 20 & 2 & C & $\mathrm{H}$ & $\mathrm{H}$ & $i$-Pr & $\mathrm{H}$ & $\mathrm{H}$ & $4 e(87)$ \\
\hline 21 & 2 & C & Et & $\mathrm{H}$ & $\mathrm{H}$ & $\mathrm{H}$ & $\mathrm{Et}$ & $\mathbf{4 f}(97)^{22}$ \\
\hline 22 & 2 & $\mathrm{C}$ & $\mathrm{F}$ & $\mathrm{H}$ & $\mathrm{H}$ & $\mathrm{H}$ & $\mathrm{H}$ & $4 \mathbf{g}(79)$ \\
\hline 23 & 2 & $\mathrm{C}$ & $\mathrm{H}$ & $\mathrm{H}$ & $\mathrm{F}$ & $\mathrm{H}$ & $\mathrm{H}$ & 4h (63) \\
\hline 24 & 2 & C & $\mathrm{Me}$ & $\mathrm{H}$ & $\mathrm{H}$ & $\mathrm{H}$ & $i-\operatorname{Pr}$ & $4 \mathbf{i}(98)$ \\
\hline 25 & 2 & C & $i-\operatorname{Pr}$ & $\mathrm{H}$ & $\mathrm{H}$ & $\mathrm{H}$ & $i-\operatorname{Pr}$ & $\mathbf{4 j}(97)^{23}$ \\
\hline 26 & 2 & C & $\mathrm{H}$ & $\mathrm{Cl}$ & $\mathrm{H}$ & $\mathrm{H}$ & $\mathrm{H}$ & $4 \mathbf{k}(88)$ \\
\hline 27 & 2 & C & $\mathrm{H}$ & $\mathrm{H}$ & $\mathrm{Cl}$ & $\mathrm{H}$ & $\mathrm{H}$ & $41(85)$ \\
\hline 28 & 2 & $\mathrm{C}$ & $\mathrm{Me}$ & $\mathrm{Cl}$ & $\mathrm{H}$ & $\mathrm{H}$ & $\mathrm{H}$ & $4 \mathbf{m}(89)$ \\
\hline
\end{tabular}

a All new compounds gave satisfactory spectroscopic and analytical data.

In conclusion, the present microwave-assisted procedure allowed a rapid parallel synthesis of a representative 28membered library of phthalimides, providing an efficient 
<smiles>[R]c1ccc2c(c1)C(=O)OC2=O</smiles><smiles>[R7][Y]1=C([R])C([R5])=[Y]([R5])C1N</smiles>

1, $\mathrm{R}=\mathrm{H}$

2, $\mathrm{R}=\mathrm{Me}$<smiles>[R][R]1c([R7])c([R7])[Y]([R7])c(N2C(=O)c3ccc([R])cc3C2=O)c1[R7]</smiles>

$$
\begin{aligned}
& \text { 3a-n }(R=H, X=C, 34-97 \%) \\
& \text { 3o (R=H, } X=N, 43 \%) \\
& \text { 4a-m (R=Me, } X=C, 63-97 \%)
\end{aligned}
$$

Scheme

methodology, with good yields, fast reaction times, and general applicability to substrates bearing a wide variety of substituents.

\section{Acnowledgement}

Authors acknowledge support of this work by the Comisión Interministerial de Ciencia y Tecnología (CICYT) through the project 2FD1997-1248.

\section{References}

(1) For some recent biological applications, see:

(a) Shimazawa, R.; Miyachi, H.; Takayama, H.; Kuroda, K.; Kato, F.; Kato, M.; Hashimoto, Y. Biol. Pharm. Bull. 1999, 22, 224. (b) Da Settimo, A.; Primofiore, G.; Da Settimo, F.; Simorini, F.; La Motta, C.; Martinelli, A.; Boldrine, E. Eur. J. Med. Chem. 1996, 31, 49. (c) Shibata, Y.; Sasaki, K.; Hashimoto, Y.; Iwasaki, S. Chem. Pharm. Bull. 1996, 44, 156. (d) Langmuir, M. E.; Yang, J.-R.; Moussa, A. M.; Laura, R.; Lecompte, K. A. Tetrahedron Lett. 1995, 36, 3989. (e) Mayer, A.; Neuenhofer, S. Angew. Chem. Int. Ed. Engl. 1994, 33, 1044. (f) Rusiecki, V. K.; Warner, S. A. Bioorg. Med. Chem. Lett. 1993, 3, 707.

(2) (a) Reddy, P. Y.; Kondo, S.; Toru, T.; Ueno, Y. J. Org. Chem. 1997, 62, 2652; and references cited therein. (b) Ohkubo, M.; Nishimura, T.; Jona, H.; Honma, T.; Morishima, H. Tetrahedron 1996, 52, 8099.

(3) Iijima, T.; Suzuki, N.; Fukuda, W.; Tomoi, M. J. Eur. Polym. $1995,31,775$.

(4) (a) Walker, M. A. J. Org. Chem. 1995, 60, 5352. (b) Walker, M. A. Tetrahedron Lett. 1994, 35, 665.

(5) Bogdal, D.; Pielichowsky, J.; Boron, A. Synlett 1996, 873.

(6) For recent papers see: (a) Vidal, T.; Petit, A.; Loupy, A.; Gedye, R. N. Tetrahedron 2000, 56, 5473. (b) Seijas, J. A.; Vazquez-Tato, P.; Martinez, M. M.; Nuñez-Corredoira, G. J. Chem. Res. (S) 1999, 420. (c) Varma, R. S.; Dahiya, R.; Kumar, S. Tetrahedron Lett. 1997, 38, 2039.
(7) (a) Chandrasekhar, S.; Padmaja, N. B.; Raza, A. Synlett 1999, 1597. (b) Chandrasekhar, S.; Takhi, M.; Uma, G. Tetrahedron Lett. 1997, 38, 8089.

(8) Borah, N. H.; Boruah, R. C.; Sandhus, J. S. J. Chem. Res.(S) 1998, 272.

(9) Parallel synthesis in microwave ovens has been described to prepare thioamide and pyridine libraries: (a) Olsson, R.; Hansen, H. C.; Andersson, C.-M. Tetrahedron Lett. 2000, 41, 7947. (b) Cotterill, I. C.; Usyatinsky, A. Y.; Arnold, J. M.; Clark, D. S.; Dordick, J. S.; Michels, P. C.; Khmelnitsky, Y. L. Tetrahedron Lett. 1998, 39, 1117.

(10) Microwave irradiation was carried out with a Sanyo microwave oven (EM-S100, 800, with a maximum power output of $800 \mathrm{~W}$ ) calibrated by a standard procedure: Watkins, K. W. J. Chem. Ed. 1983, 60, 1043.

(11) Acquired from International Sorbent Technology Ltd., U.K.

(12) Savant Automatic Environmental SpeedVac (AES 2000) was used.

(13) Microsoft $\circledast$ Excel 97 was used.

(14) Shibata, Y.; Sasaki, K.; Hashimoto, Y.; Iwasaki, S. Chem. Pharm. Bull. 1996, 44, 156.

(15) Kamal, A.; Laxman, E.; Laxman, N.; Rao, V. Tetrahedron Lett. 1998, 39, 8733.

(16) Pagani, G.; Baruffini, A.; Borgna, P.; Caccialanza, G. Farmaco, Ed. Sci. 1968, 23, 448; Chem. Abstr. 1969, 69, 43588.

(17) Miyachi, H.; Azuma, A.; Ogasawara, A.; Uchimura, E.; Watanabe, N.; Kobayashi, Y.; Kato, F.; Kato, M.; Hashimoto, Y. J. Med. Chem. 1997, 40, 2858.

(18) Fifolt M. J., Sojka S. A., Wolfe R. A., Hojnicki D. S., Bieron J. F., Dinan F. J.; J. Org. Chem.; 1989, 54: 3019.

(19) Abdel-Rahman, A. E.; Khalil, Z. H. Bull. Chem. Soc. Jpn. 1978, 51, 2148.

(20) Krische, M. J.; Lehn, J. M.; Kyritsakas, N.; Fisher, J. Helv. Chim. Acta 1998, 81, 1909.

(21) Perry, R. J.; Turner, R. J. Org. Chem. 1991, 56, 6573.

(22) Hokko Chemical Industry Co. Ltd., Jpn. Kokai Tokkyo Koh, 1980, Jp55136210A2; Chem. Abstr. 1980, 94, 78435.

(23) Hasimoto, Y. PCT Int. Appl. WO9807421, 1998; Chem. Abstr. 1998, 128, 204894.. 